\title{
Prevalence of urinary incontinence in a cohort of women with obesity
}

\author{
Giulia Trotti ${ }^{\mathrm{a}}$, Valentina Aspesi ${ }^{\mathrm{a}}$, Lorena De Ambroggi ${ }^{\mathrm{b}}$, Veronica Cimolin ${ }^{\mathrm{c}}$, Ionathan Seitanidis ${ }^{\mathrm{a}}$, \\ Luigia Brugliera $^{\mathrm{d}}$ and Paolo Capodaglio ${ }^{\mathrm{a}, \mathrm{e}, *}$ \\ ${ }^{a}$ Istituto Auxologico Italiano, IRCCS, Rehabilitation Unit and Research Lab in Biomechanics and Rehabilitation, \\ Piancavallo, Italy \\ ${ }^{\mathrm{b}}$ Istituti Clinici Scientifici Maugeri Spa SB (IRCCS), Istituto Scientifico di Veruno, Veruno, Italy \\ ${ }^{\mathrm{c}}$ Department of Electronics, Information and Bioengineering, Politecnico di Milano, Milano, Italy \\ ${ }^{\mathrm{d}}$ Department of Rehabilitation and Functional Recovery, IRCCS San Raffaele Scientific Institute, Vita-Salute \\ University, Milan, Italy \\ ${ }^{\mathrm{e}}$ Physical Medicine and Rehabilitation, Department of Surgical Sciences, University of Turin, Turin, Italy
}

Received 12 October 2020

Accepted 28 June 2021

\begin{abstract}
.
INTRODUCTION: Urinary incontinence (UI) is frequently associated with obesity. The prevalence of the different UI types in women with obesity remains scarcely investigated and controversial.

OBJECTIVE: The goal of this study was to investigate the prevalence of the different types of UI (stress urinary incontinence, SUI, urge, UUI, or mixed, MUI) in a large sample of female patients with obesity by means of a specific questionnaire and non-invasive tests.

METHODS: In this observational study, 248 obese female patients (BMI $\geq 30 \mathrm{Kg} / \mathrm{m} 2$, age: $62.8+10.9$ years) admitted to hospital from April 2019 to September 2019 for a multidisciplinary rehabilitation program were recruited for this study. The International Consultation on Incontinence Questionnaire - short form (ICIQ-sf) was used to screen the presence of symptoms of UI and to differentiate the different UI types. Patients with ICIQ-sf score $\geq 4$, were asked to undertake the Pad Test for quantifying urine leaks under stress.

RESULTS: $61.69 \%$ of our sample presented UI symptoms. The prevalence of UI appears to be lower in younger age groups (57\% in 31-46 years of age and 52\% in 47-62 years of age) and higher (69\%) between 63 and 79 years of age. MUI was the most frequent form $(57.5 \%)$, followed by UUI $(21.5 \%)$ and SUI (20.9\%). SUI was most prevalent in younger participants (31-46 years old).

CONCLUSION: This study demonstrated that UI has a high prevalence in females with obesity and it is not an exclusive concern of older women. This high prevalence calls for specific rehabilitation interventions within multidisciplinary programs.
\end{abstract}

Keywords: Urinary incontinence, prevalence, obesity, quality of life, rehabilitation

\section{Introduction}

The International Continence Society recognises three forms of Urinary Incontinence (UI) [1, 2]: a) stress urinary incontinence (SUI), when the urinary leak is caused by a stimulus like sneeze, physical

\footnotetext{
*Corresponding author: Paolo Capodaglio, MD, Istituto Auxologico Italiano, IRCCS, Rehabilitation Unit and Research Lab in Biomechanics and Rehabilitation, Str. L Cadorna, 90, 28824, Piancavallo, VB, Italy. E-mail: p.capodaglio@auxologico.it.
}

stress or cough that cause pressure to the bladder; b) urge urinary incontinence (UUI), determined by a sudden and incontrollable contraction of the bladder; c) mixed urinary incontinence (MUI), the combination of the two previous types.

A recent internet survey based on a health-related quality of life questionnaire [3] on a sample of 8284 subjects over 40 years of age in China, Taiwan and South Korea showed a general prevalence of UI of $22 \%$ (17,3\% in males, $26,4 \%$ in females). The most 
frequent UI type appears to be MUI $(9,7 \%)$, followed by SUI $(7,9 \%)$ and UUI $(4,3 \%)$.

Women are more prone to UI than men in every age range $[4,5]$. This is due to a combination of factors, including female anatomy, hormonal changes and pregnancy, which can cause relaxation of the pelvic floor and consequent urinary leaks' $[6,7]$. Obesity is strongly associated with UI, independently from other factors $[1,8,9]$ : the risk of developing UI increases $20 \%$ to $70 \%$ every 5-unit BMI increase [9]. A prevalence of $60 \%$ has been previously reported in women with obesity $[10,11]$. From a pathophysiological point of view, increases in intra-abdominal pressure secondary to obesity lead to an increase in bladder pressure and stress on the pelvic muscles thus determining UI [12]. However, there is limited conflicting research on the prevalence of the three different UI types in women with obesity. Three studies reported SUI to be more frequent as compared to UUI (25\% vs $15 \%$ [13] and $30 \%$ vs $15 \%[14,15]$ ). Another study reported MUI as the most frequent type [11].

UI has an impact on social, personal hygiene and Quality of life (Qol) aspects [16-18].

Since both UI and obesity can lead to a reduced Qol and high costs for National Health Systems, it is therefore important to investigate the relationship of these two factors. UI should be diagnosed on the basis of laboratory findings, bladder function, imaging tests together with clinical examination and bladder diary.

When the aim is to assess the presence of UI in large cohorts of subjects with obesity the whole range of tests and evaluations can be unpractical and not always feasible to be carried out. For epidemiological purposes questionnaires for the detection of UI symptoms have been developed. The goal of the present study was therefore to investigate the prevalence of the different types of UI (SUI, UUI or MUI) in a large sample of female patients with obesity by means of a specific questionnaire and non-invasive tests.

\section{Materials and methods}

\subsection{Participants}

From April 2019 to September 2019, we consecutively enrolled into this study 248 female patients with $\mathrm{BMI} \geq 30 \mathrm{Kg} / \mathrm{m}^{2}$ and with an age between 30 and 79 years, who had been admitted to S Giuseppe Hospital, Istituto Auxologico Italiano, for a multidisciplinary rehabilitation program. Exclusion criteria were: anterior prolapse greater than 3rd degree, inability to walk for 30 meters, presence of neurological bladder, psychiatric conditions or dementia that would hamper the use of questionnaires. The study was approved by the Ethical Committee of the Istituto Auxologico Italiano. All procedures performed in the study were in accordance with the ethical standards of the institutional and national research committee and with 1964 Declaration of Helsinki and its later amendments or comparable ethical standards. All participants gave written informed consent after being fully informed about the purpose of the study and the option to withdraw at any time.

\subsection{Outcome measures}

The International Consultation on Incontinence Questionnaire - short form (ICIQ-sf) was used to assess the presence of UI symptoms. The ICIQ-sf evaluates frequency, severity and impact of UI on quality of life (Qol). The four parameters investigated were: frequency and quantity of the leaks, global impact of UI and overall patient's condition [19]. The questionnaire also investigates the different UI types by asking whether leaks occur after coughing or physical activity (SUI), or before managing to get to the toilet on time (UUI), or a combination of the two (MUI). The score ranges from 0 to 21 points, where 1-5 = mild UI; $6-12=$ moderate UI; $13-18=$ severe UI; 19-21 = very severe UI [20]. A total ICIQ-sf score greater than or equal to 4 was considered positive for UI.

Patients who resulted positive for SUI or MUI underwent the 1-hour Pad Test, a first-level urodynamic test to quantify urinary leaks [21]. This provocative test consists of asking the patient to wear a dry diaper that had been previously weighted, then drink $500 \mathrm{ml}$ of water, and, after $30 \mathrm{~min}$, perform various physical activities (walk, climb stairs, raise from a sitting position for 10 times, cough for 10 times, run on the spot for a minute, bend down and lift an object from the ground for five times) while wearing the pad. The total duration of the test is one hour. At the end of the test, the diaper is weighted again and if the weight variation is $\geq 1 \mathrm{~g}$ the test is considered positive for SUI [16].

The Statistica software (statSoft, USA) was used for statistical analysis.

The analysis followed a qualitative description and quantitative presentation of the findings using frequency distribution, percentages, and graphs. Then, the mean value and standard deviation of ICIQ-sf score and the data of the group of patients less than 65 
Table 1

Demographic features of the analyzed sample (mean and standard deviation)

\begin{tabular}{lc}
\hline & Mean and standard deviation \\
\hline Age $(\mathrm{yrs})$ & $62.8(10.9)$ \\
BMI $\left(\mathrm{Kg} / \mathrm{m}^{2}\right)$ & $44.8(7.1)$ \\
ICIQ-sf score & $7.4(6.2)$
\end{tabular}

BMI: Body Mass Index; ICIQ-sf: International Consultation on Incontinence Questionnaire - short form.

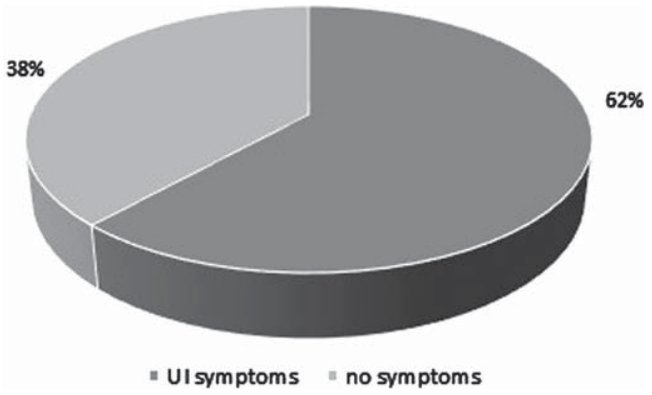

Fig. 1. Patients (number and percentage) with and without symptoms of urinary incontinence (UI)

and over 65 years were compared with $t$-test for independent samples. The Pearson correlation coefficient was calculated to examine the relationship between ICIQ-sf score and BMI. The level of significance was set at $p=0.05$, as this is a widely accepted criterion for clinically meaningful evidence in research, providing a strong evidence against the null hypothesis [22].

\section{Results}

The demographic features of the 248 participants are described in Table 1 . The age range of participants was $31-79$ years (mean age: $62.8+10.9$ years). BMI ranged from $30.5 \mathrm{Kg} / \mathrm{m}^{2}$ to $72.7 \mathrm{Kg} / \mathrm{m}^{2}$. The number of participants with UI (ICIQ-sf score $\geq 4$ ) was 153/248 (61.7\%) (Fig. 1).

MUI (57.5\%) was the most prevalent type of UI, followed by UUI $(21.6 \%)$ and then SUI $(20.9 \%)$ (Fig. 2).

We reported the mean values of the scores obtained in the ICIQ-sf questionnaire of participants aged under 65 years and over 65 years (Table 2). From the data it is possible to observe that the difference between under and over 65 years is statistically different in terms of ICIQ-sf score $(p<0.05)$.

The prevalence of UI was lower in the younger patients (prevalence of 57\% in the age range 31-46

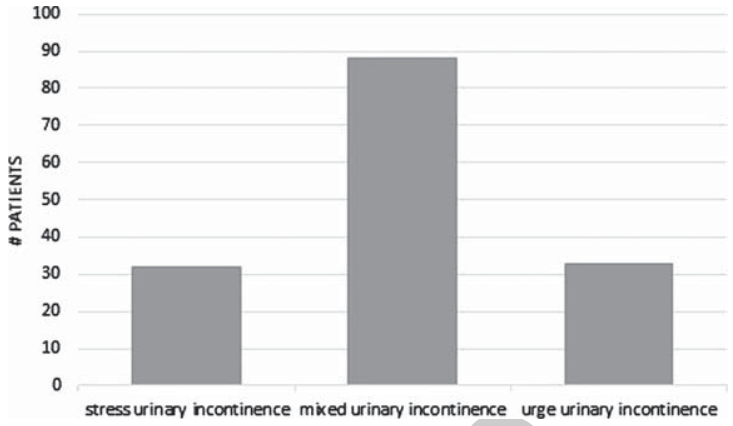

Fig. 2. The three different types of UI.

Table 2

Mean (standard deviation) of ICIQ-sf score for patients under and over 65 years. ${ }^{*}=p<0.05$, under 65 years vs. over 65 years

\begin{tabular}{lcc}
\hline & Under 65 years & Over 65 years \\
\hline Numerosity (\#) & 138 & 110 \\
ICIQ-sf score & $5.9(5.6)$ & $9.4(6.4)^{*}$ \\
\hline
\end{tabular}

ICIQ-sf: International Consultation on Incontinence Questionnaire - short form.

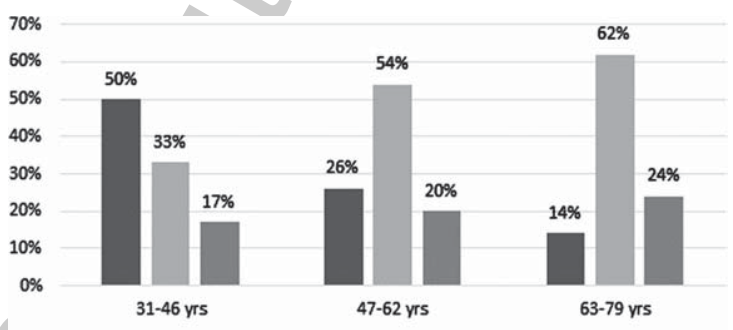

m stress urinay incontinence $=$ mixed urinary incontinence $\|$ urge urinary incontinence

Fig. 3. Different types of UI in different age ranges.

years, and $52 \%$ in the age range $47-62$ years) as compared to older patients (prevalence of $69 \%$ in the age range 63-79 years).

SUI was the most prevalent type of UI $(50 \%)$ in younger participants (31-46-year-old), followed by MUI (33\%) and UUI (17\%). In the other two age ranges (47-62 and 63-79-year-old patients) the prevalence of SUI decreased, while MUI and UUI increased parallel to age: in 47-62-year-old patients SUI was $26 \%$, MUI $54 \%$, UUI $20 \%$; in 63-79-yearold patients SUI was $14 \%$, MUI $62 \%$, UUI $24 \%$ (Fig. 3).

In our study, no correlation was found between different BMI and the severity of incontinence (ICIQsf scores) $(p>0.05)$.

Out of 153 patients with UI symptoms (ICIQ-sf score $\geq 4), 120$ who presented stress incontinence symptoms (SUI or MUI) undertook the 1-Hour 
Pad Test; $55 \%$ of participants presented with urine leaks $\geq 1 \mathrm{~g}$, evidencing the presence of SUI.

\section{Discussion}

In subjects with obesity an increase in the loading on the pelvic muscles and on the bladder has been reported [23]. A $60 \%$ prevalence of UI has been reported in the literature. Our general prevalence data $(61.69 \%)$ are in line with the current literature. None of our experimental subjects withdrew from the study. UI appears to be relatively less prevalent in younger age groups, whereas the prevalence rises to $69 \%$ in the age range between 63 and 79 years. According to our findings, the most frequent type of UI in our experimental sample with obesity was MUI (57.5\%), followed by the SUI (21.5\%) and UUI (20.9\%).

MUI typically appears after menopause and can be attributed to the decrease of blood estrogens level after menopause, causing urogenital atrophy and development of lower urinary tract infections facilitating urinary leaks, a hyperactivity of the detrusor muscle of the bladder and alteration of the tissue trophism [24]. Our findings of a UI prevalence of $57 \%$ in $31-46$ years old and of 52\% in 47-62 years old women suggest that UI does not appear a problem exclusive of elderly women.

The novelty of our study was to describe the prevalence of the three UI types across different ages. As for which UI type was more prevalent in different age ranges, SUI was reported in 50\% of the younger (31-46-year-old) patients, followed by MUI (33\%) and UUI (17\%). The excess of body mass induces an increase in intra-abdominal pressure with consequent overload of ligaments and muscles of the pelvic floor, ultimately determining SUI in younger patients. In the other two age ranges (47-62 and 63-79-yearold patients), the prevalence of SUI decreased, while MUI and UUI increased parallel to age. In the older patients, factors related to hormonal changes due to menopause may account for the onset of UUI (i.e., hyperactivity of the detrusor muscle), which eventually may evolve into MUI.

UI-related reduction in Qol and discomfort in daily and sexual life may therefore occur also in younger patients with obesity $[25,26]$. UI may also worsen depression and anxiety that are frequent obesity-related features, thus negatively impacting the patients' social and productive life [27, 28].

The questionnaire we used in this study, the ICIQ$\mathrm{SF}$, was developed for a clinical use on all patients with UI regardless of gender and age and for comparing studies on UI. It is a brief and psychometrically robust patient-completed questionnaire for evaluating frequency, severity and impact on Qol of UI in research and clinical practice across the world. It includes 3 questions related to the frequency of UI, the ordinary amount of UI, and its influence on daily life. The scores of the 3 questions were summed and the resulting totals ranged between 0 and 21 points.. The validity, reliability, and responsiveness of the ICIQ-SF for UI had been previously verified [19].

Out of 153 patients with UI symptoms (ICIQ-sf score $\geq 4$ ) on a total sample of 248,120 who presented stress incontinence symptoms (SUI or MUI) undertook the 1-Hour Pad Test. Only 55\% of the patients presenting stress incontinence symptoms were positive at the 1 -Hour Pad Test with urine leaks $\geq 1 \mathrm{~g}$. Several factors may have accounted for the discrepancy between subjective perception of the symptoms, as assessed by the questionnaire, and the quantification of urine leaks, as assessed by the 1Hour Pad Test. Individual emotional and behavioral factors, different levels of physical activity and different bladder filling durations among the experimental subjects, which were not investigated in this study, may have indeed affected the results. Since individual habitual levels of physical capacity were not known, the stresses imposed during the 1-Hour Pad Test may have not been sufficient for all the patients to provoke urinary leaks. The test is non-invasive and easy to perform, yet factors such as embarrassment and behavioral changes to reduce incontinence severity (inactivity, fluid restriction) could significantly affect the outcome. Also, variability in how the test has been performed in different studies makes comparison of published results difficult. Different testing durations have been reported in the literature, but only for the 1-hour Pad Test a specific test protocol has been standardized [29, 30]. A number of studies have documented that the longer the testing, the better the correlation between the test results and the degree of incontinence. However, 24- to 72-hr Pad Tests are cumbersome and require high levels of patient compliance [31]. For such reasons, we used the 1hour Pad Test. We are aware that negative 1-hour Pad Test should be cautiously interpreted. Unfortunately, organizational constraints did not allow repeated short-term testing in cases where test result did not correlate with subjective assessment provided by the patient. This could also have accounted for the discrepancy observed between subjective complaints 
of incontinence symptoms (questionnaire) and their objective measurement (Pad Test).

Our study presents with other limitations. Our patients did not have a clinical and instrumental urodynamic diagnosis. The ICIQ-sf questionnaire used in this study does not define cut-off values to establish whether or not a patient presents UI symptoms. For this reason, we opted for a cut-off value of 4 points, which represents the minimum score associated with UI symptoms.

Another limitation was that the presence of residual urine in the bladder was not investigated and, consequently, urinary retention was not considered as a possible cause of UI. However, urinary retention is usually secondary to neurological disorders causing dyssynergia and premature closure of the urethral sphincter, which were among the exclusion criteria of the study.

In conclusion, data emerging from this observational study about the association between obesity and UI are in line with the literature. The experimental sample of female patients with obesity in our study shows a wide age range and appears representative of a general population with obesity. The high prevalence of UI calls for specific interventions within multidisciplinary rehabilitation programs. UI is not only a feature of older age, it also affects younger women with obesity and their Qol. Further research is needed to investigate whether rehabilitation interventions for the pelvic floor are effective in the obese population and can be age- and symptom-specific.

\section{Conflict of interest}

The authors have no conflict of interest to report.

\section{References}

[1] Abrams P, Cardozo L, Wagg A, Wein A. Incontinence. 6th ed. Tokyo: Icud; 2017

[2] Haylen BT, de Ridder D, Freeman RM, Swift SE, Berghmans B, Lee J, Monga A, Petri E, Rizk DE, Sand PK, Schaer GN; International Urogynecological Association; International Continence Society. An International Urogynecological Association (IUGA)/International Continence Society (ICS) joint report on the terminology for female pelvic floor dysfunction. Neurourol Urodyn. 2010;29(1): 4-20.

[3] Sumarsono B, Jong JJ, Wang JY, Liao L, Lee KS, Yoo TK, et al. The prevalence of urinary incontinence in men and women aged 40 years or over in China, Taiwan and South Korea: A cross-sectional, prevalence-based study. Low Urin Tract Symptoms 2020 Mar 23
[4] Aoki Y, Brown HW, Brubaker L, Cornu JN, Daly JO, Cartwright R. Urinary incontinence in women. Nat Rev Dis Primers. 2017;3:17042.

[5] Wiethaler V. Il pavimento pelvico: come rivitalizzarlo e rinforzarlo. Perugia: Morlacchi Editore; 2007.

[6] Calleja-Agius J, Brincat MP. The urogenital system and the menopause. Climacteric. 2015;18(Suppl 1):18-22.

[7] Rocha J, Brandão P, Melo A, Torres S, Mota L, Costa F. Avaliação da Incontinência Urinária na Gravidez e no Pós-Parto: Estudo Observacional [Assessment of Urinary Incontinence in Pregnancy and Postpartum: Observational Study]. Acta Med Port. 2017;30(7-8):568-72.

[8] Fuselier A, Hanberry J, Lovin JM, Gomelsky A. Obesity and Stress Urinary Incontinence: Impact on Pathophysiology and Treatment. Current Urology Reports. 2018;19(1):10.

[9] Subak LL, Richter HE, Hunskaar S. Obesity and urinary incontinence: epidemiology and clinical research update. $\mathrm{J}$ Urol. 2009;182(6):2-7.

[10] Richter HE, Burgio KL, Clements RH, Goode PS, Redden DT, Varner RE. Urinary and anal incontinence in morbidly obese women considering weight loss surgery. Obstet Gynecol. 2005;106(6):1272-7.

[11] Schreiber Pedersen L, Lose G, Høybye MT, Elsner S, Waldmann A, Rudnicki M. Prevalence of urinary incontinence among women and analysis of potential risk factors in Germany and Denmark. Acta Obstet Gynecol Scand. 2017;96(8):939-48.

[12] Marcelissen T, Anding R, Averbeck M, Hanna-Mitchell A, Rahnama'i S, Cardozo L. Exploring the relation between obesity and urinary incontinence: Pathophysiology, clinical implications, and the effect of weight reduction, ICI-RS 2018. Neurourol Urodyn. 2019;5:S18-S24.

[13] Brucker J, Wagner I, Rudofsky G, Rauch G, Sohn C, Brocker KA. In obesity even young women suffer from urogynecological symptoms. Arch Gynecol Obstet. 2017;296(5): 947-56.

[14] Townsend MK, Danforth KN, Rosner B, Curhan GC, Resnick NM, Grodstein F. Body mass index, weight gain, and incident urinary incontinence in middle-aged women. Obstet Gynecol. 2007;110(2 Pt 1):346-53.

[15] Waetjen LE, Liao S, Johnson WO, Sampselle CM, Sternfield B, Harlow SD, et al. Factors associated with prevalent and incident urinary incontinence in a cohort of midlife women: a longitudinal analysis of data: study of women's health across the nation. Am J Epidemiol. 2007;165(3):309-18.

[16] Bortolami A. Riabilitazione del pavimento pelvico. Edra S.p.A. Milano; 2017

[17] Subak, LL, Brubaker L, Chai TC, Creasman JM, Diokno AC, Goode PS, et al. High costs of urinary incontinence among women electing surgery to treat stress incontinence. Obstet. Gynecol. 2008;111 (4):899-907.

[18] Melville JL, Delaney K, Newton K, Katon W. Incontinence severity and major depression in incontinent women. Obstet Gynecol. 2005;106(3):585-92.

[19] Avery K, Donovan J, Peters T, Shaw C, Gotoh M, Abrams P. ICIQ: a brief and robust measure for evaluating the symptoms and impact of urinary incontinence. Neurourol Urodyn. 2004;23(4):322-30.

[20] Klovning A, Avery K, Sandvik H, Hunskaar S. Comparison of two questionnaires for assessing the severity of urinary incontinence: The ICIQ-UI SF versus the incontinence severity index. Neurourol Urodyn. 2009;28(5):411-5.

[21] Abrams P, Andersson KE, Birder L, Brubaker L, Cardozo L, Chapple C, et al. Fourth International Consultation on Incontinence Recommendations of the International 
Scientific Committee: Evaluation and treatment of urinary incontinence, pelvic organ prolapse, and fecal incontinence. Neurourol Urodyn. 2010;29:213-40.

[22] NA Weiss. Introductory Statistics, 9th Edition, 2012, Pearson Publisher

[23] Swenson CW, Kolenic GE, Trowbridge ER, et al. Obesity and stress urinary incontinence in women: compromised continence mechanism or excess bladder pressure during cough? Int Urogynecol J Pelvic Floor Dysfunct. 2017; 28(09):1377-85.

[24] Faubion SS, Sood R, Kapoor E. Genitourinary Syndrome of Menopause: Management Strategies for the Clinician. Mayo Clin Proc. 2017;92(12):1842-9.

[25] Wyman JF, Harkins SW, Choi SC, Taylor JR, Fantl JA. Psychosocial impact of urinary incontinence in women. Obstet Gynecol. 1987;70(3 Pt 1):378-81.

[26] Verbeek M, Hayward L. Pelvic Floor Dysfunction And Its Effect On Quality Of Sexual Life. Sex Med Rev. 2019;7(4):559-64. doi: 10.1016/j.sxmr.2019.05.007. Epub 2019 Jul 24

[27] Tang DH, Colayco DC, Khalaf KM, Piercy J, Patel V, Globe $\mathrm{D}$, Ginsberg D. Impact of urinary incontinence on healthcare resource utilization, health-related quality of life and productivity in patients with overactive bladder. BJU Int. 2014;113(3):484-91.
[28] Minassian VA, Sun H, Yan XS, Clarke DN, Stewart WF. The interaction of stress and urgency urinary incontinence and its effect on quality of life. Int Urogynecol J. 2015;26(2): 269-76.

[29] Seventh report on the standardization of terminology of lower urinary tract function: Lower urinary tract rehabilitation techniques. International Continence Society Committee on Standardisation of Terminology. Scand J Urol Nephrol. 1992;26:99-106.

[30] Persson J, Bergqvist CE, Wolner-Hanssen P. An ultra-short perineal pad-test for evaluation of female stress urinary incontinence treatment. Neurourol Urodyn. 2001;20: 277-85.

[31] Zimmern P, Kobashi K, Lemack G. Outcome measure for stress urinary incontinence treatment (OMIT): Results of two society of urodynamics and female urology (SUFU) surveys. Neurourol Urodyn. 2010;29:715-8. 